\title{
Life threatening airway obstruction secondary to a large probable parathyroid cyst.
}

\author{
K McCullough; V Constantinidis; M Badman, J Jackson, F Palazzo. \\ Imperial Centre for Endocrinology, Hammersmith Hospital, Imperial College Healthcare NHS Trust, Du Cane Road, \\ London, W12 0NN.
}

\begin{abstract}
Parathyroid cysts are rare, usually asymptomatic and typically present as a neck lump. They are most commonly detected in middle-aged women and can occasionally present with compressive symptoms. True parathyroid cysts are nonfunctional and benign in nature, allowing a more conservative approach to their management in many patients. We present the case of an 82 year old lady with a past medical history of a presumed parathyroid cyst which was drained by the ENT surgeons. Three years later, she presented to hospital with sudden onset shortness of breath, throat pain and three months of increasing dysphagia. Examination and initial investigations were consistent with upper airway obstruction and a chest radiograph showed a large retrosternal mass. Computed tomography revealed a large $8 \times 5 \mathrm{~cm}$ cyst causing tracheal compression and deviation. The patient underwent drainage of the cyst via ultrasound and fluoroscopic guidance with full resolution of symptoms. Histology and intra-cystic PTH levels were consistent with a large parathyroid cyst however an alternative diagnosis of a $3^{\text {rd }}$ branchial arch cyst could not be excluded. We discuss the prevalence and presentation of such cysts and different management options. This case illustrates a rare, yet important cause of a neck lump that can present with life-threatening upper airway obstruction.
\end{abstract}

\section{CASE REPORT}

An 82 year old lady presented to the Emergency Department with sudden onset shortness of breath, throat pain and a three month history of increasing dysphagia. Three years earlier she presented to the Ear, Nose and Throat surgeons with increasing dysphagia and was found to have a large presumed parathyroid cyst. At that time, she underwent percutaneous drainage with resolution of symptoms (cystic PTH 80 pmol/l, serum PTH $1.2 \mathrm{pmol} / \mathrm{l}$ ).

On this admission, she presented to the Emergency Unit with audible stridor, respiratory rate of 24 , reduced air entry throughout both lung fields and distended neck veins. There was no palpable goitre. Oxygen saturations were $98 \%$ with a pO2 of $32 \mathrm{kPa}$ on $10 \mathrm{~L}$ oxygen. Peak expiratory flow rate was $60 \mathrm{~L} / \mathrm{min}$. Routine blood tests including thyroid function and calcium studies were normal. A chest radiograph revealed a large retrosternal mass (fig.1). Initial treatment included intravenous dexamethasone, adrenaline nebulisers and heliox gas.

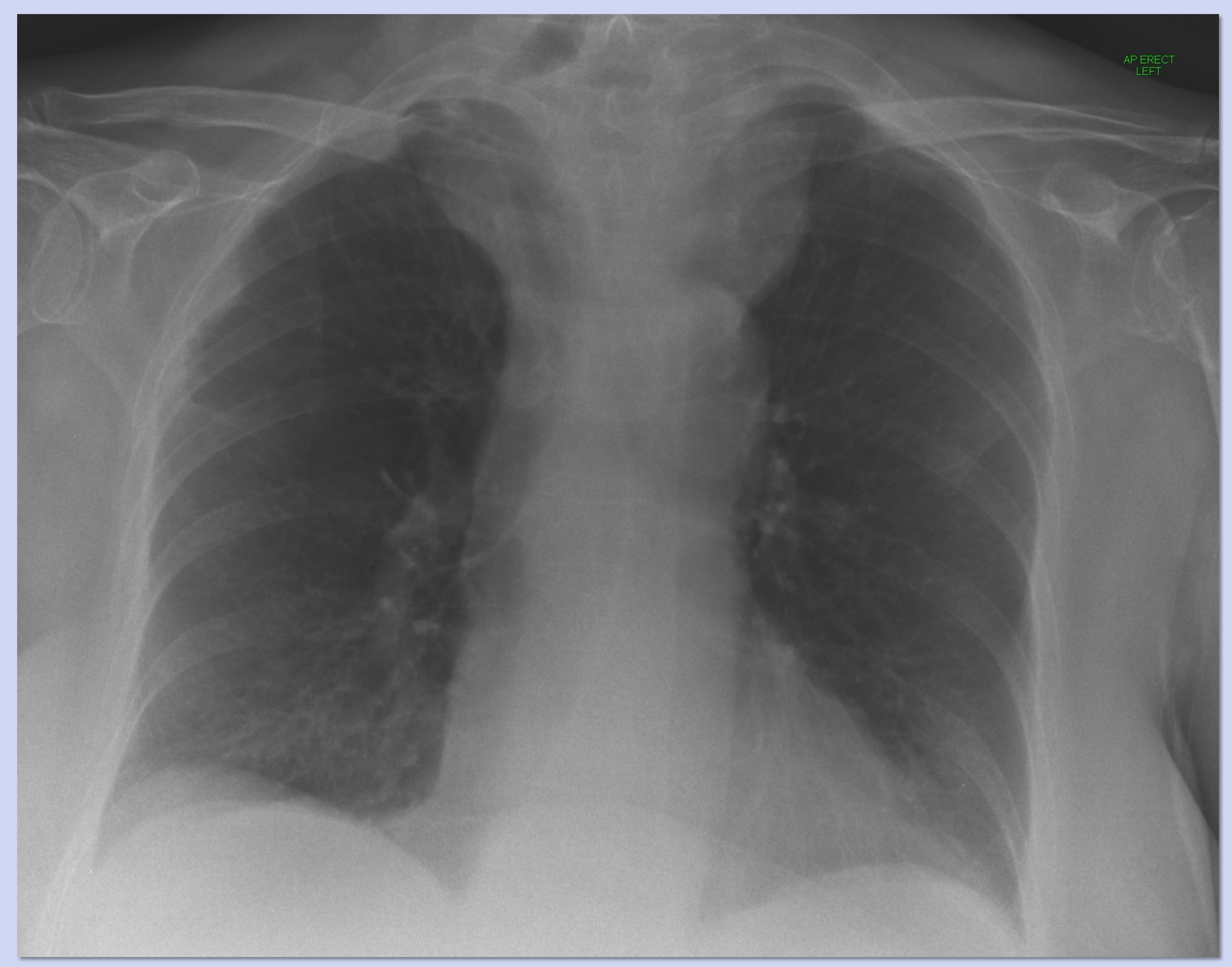

Figure 1. Chest radiograph demonstrating large retrosternal soft tissue mass causing considerable tracheal deviation towards the right.
A CT neck and thorax was performed and demonstrated an $8 \times 5 \mathrm{~cm}$ cystic mass in the superior mediastinum which looked to be continuous with the left lobe of the thyroid gland. In addition, this caused considerable deviation of the trachea towards the right side and narrowed its coronal diameter to $5 \mathrm{~mm}$ (fig.2). The cyst was punctured percutaneously under ultrasound guidance and $100 \mathrm{mls}$ of clear fluid was aspirated and sent for laboratory analysis (cystic PTH $12.3 \mathrm{pmol} / \mathrm{l}$, serum PTH $4.1 \mathrm{pmol} / \mathrm{l})$. Her symptoms completely resolved and she was discharged home the next day.

Tracheal compression

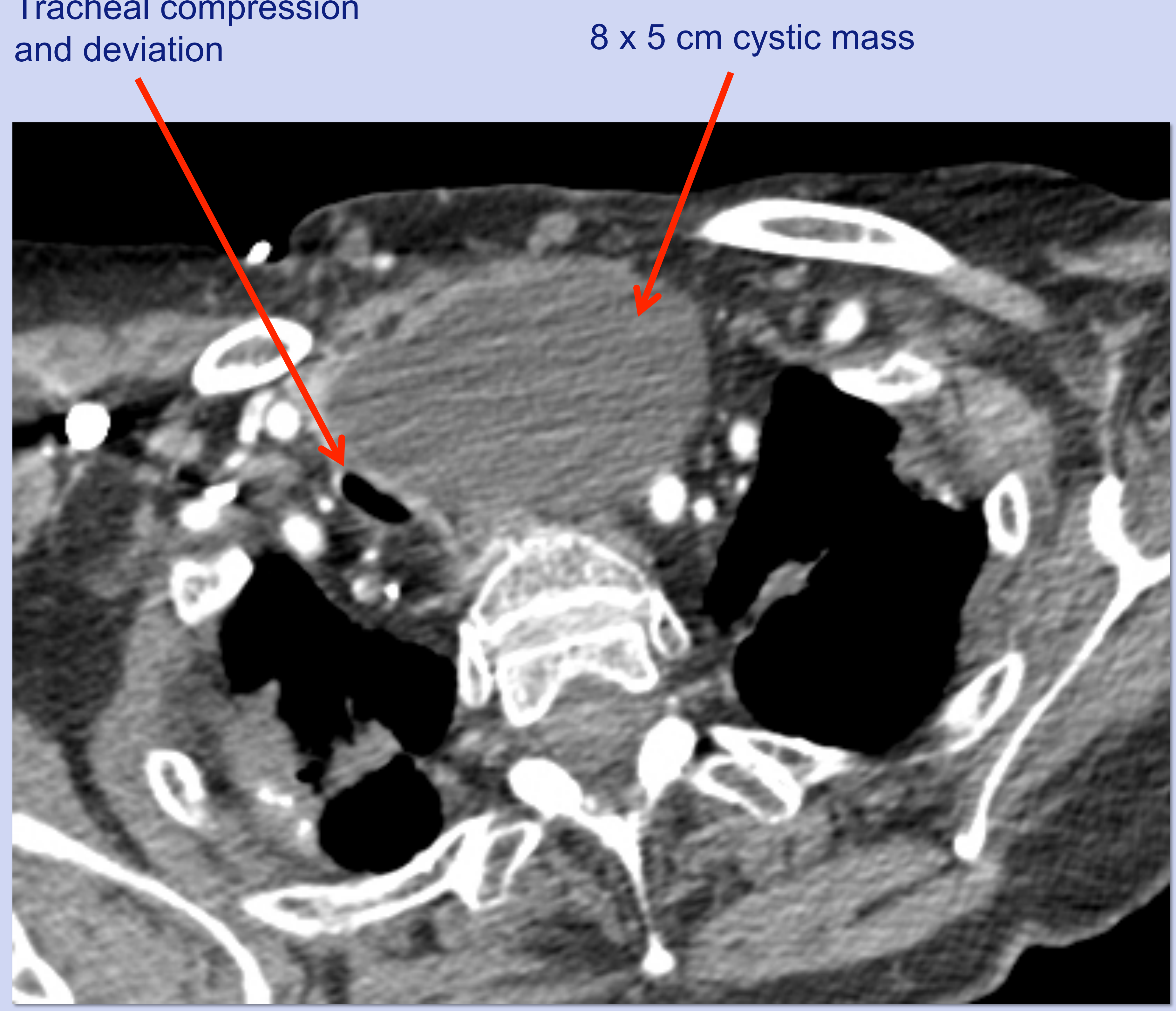

Figure 2. CT thorax demonstrating an $8 \times 5 \mathrm{~cm}$ cystic mass causing right-sided tracheal deviation and compression.

\section{DISCUSSION}

This case report describes the presentation of a large parathyroid cyst causing marked upper airway obstruction. Parathyroid cysts are rare with a reported incidence amongst patients having had thyroid and parathyroid surgery of $<1 \%$ (1). Presentation is usually incidental following a chest radiograph, compressive symptoms or palpable neck mass. Initial management of parathyroid cysts may include aspiration or surgical resection. Parathyroid cysts are characterised by the appearance of the fluid (clear) and high cystic PTH concentration. In one case series describing 38 parathyroid cysts, the size of the cysts ranged from $10-60 \mathrm{~mm}$ with intra-cystic PTH values ranging from $9-319 \mathrm{pmol} / \mathrm{l}$, with intra-cystic fluid volume ranging from 10-40 mls clear fluid.

In the case described here, the presence of clear fluid and high levels of PTH following her first presentation are strongly suggestive of a parathyroid cyst $(2,3)$. Despite the high concentration of PTH found within true parathyroid cysts, they are non-functional and therefore patients have normal circulating calcium and PTH levels. The origin of parathyroid cysts are unknown although it has been suggested that they likely represent embryological remnants and are of branchial origin (1). Therefore, in this case report, it is likely that this lady presented with a parathyroid cyst, although a $3^{\text {rd }}$ branchial arch cyst cannot be excluded.

\section{CONCLUSION}

This case describes the presentation of a large probable parathyroid cyst presenting with tracheal compression. Parathyroid cysts are rare, yet an important cause of a neck lump that can present with life-threatening upper airway obstruction. 